\title{
Linear and nonlinear behavior in single-ring resonators
}

\author{
Abdolkarim Afroozeh ${ }^{1}$
}

Received: 22 May 2018/ Accepted: 13 June 2018/Published online: 19 June 2018

(C) The Author(s) 2018

\begin{abstract}
This study has been focused on the linear and nonlinear behavior and investigative in single-ring resonator. Optical fiber ring resonators have great interest due to different applications, especially in communication, secured communication, slow light, fast light and light storage in optical buffers. There are many proposed applications of optical ring resonators. Optical fiber ring resonators can be readily constructed from standard optical fiber components, which allow their properties to be studied in a systematic manner. In this paper, we describe our studies of the optical transmission, intensity, shift phase and group delay characteristics of a family of such devices. In this case, Gaussian beam has been used as an input pulse inside to microring resonators. In this paper, transmission, intensity, shift phase and group delay characteristics of device have been investigated. It has been compared together in different coupling coefficients in frame of linear and nonlinear.
\end{abstract}

Keywords Transmission $\cdot$ Intensity $\cdot$ Shift phase $\cdot$ Group delay $\cdot$ Optical fiber ring resonator

\section{Introduction}

There are many proposed applications of optical ring resonators [1-5]. These devices can be constructed in a variety of sizes from micrometers to nanometers, with spectral characteristics that scale. Optical fiber ring resonators [6-8] can be readily constructed from standard optical fiber components, which allow their properties to be studied in a systematic manner. In this paper, we describe our studies of the optical transmission, intensity, shift phase and group delay characteristics of a family of such devices. Optical ring resonators hold great promise for a variety of applications, including optical switching [9, 10], optical time delay [10-12], photonic biosensors [3, 13, 14], laser resonators [1] and add-drop filters [15, 16].

\section{Analysis of optical fiber ring resonator (OFRR)}

Optical fiber ring resonators have great interest due to different applications, especially in communication and light storage in optical buffers. In this work, optical fiber

Abdolkarim Afroozeh

afroozeh@lar.ac.ir

1 Department of Engineering, University of Larestan, Lar, Iran has been made form InGaAsP/InP [17-19]. The schematic diagram of an optical fiber ring resonator system is shown in Fig. 1. The fiber coupler is connected to one ring of the resonator system. The nonlinearity of the fiber ring is of the Kerr type. The refractive index of the proposed system is given by Eq. (1)

$n=n_{0}+n_{2} I=n_{0}+\left(\frac{n_{2}}{A_{\mathrm{eff}}}\right) P$,

where $n_{2}$ is the nonlinear refractive index and $n_{0}$ is the linear refractive index. $I$ and $P$ are the optical intensity and optical field power, respectively. $A_{\text {eff }}$ is the effective mode core area of the fiber. The study of nonlinear phenomena in fiber ring resonators plays an important role in optical communication. The nonlinearity leads to a decrease in the line width and absorption. Therefore, these phenomena assist the generation of slow light in microring resonator. For simplification of the equation, the output field at steady state is given as:

$$
\begin{aligned}
y_{1} & =\sqrt{1-\kappa_{1}}, x_{1}=\sqrt{1-\gamma_{1}}, \tau=\exp (-\alpha L / 2), \phi \\
& =k L n_{0}+k L n_{2}\left|E_{1}\right|^{2}
\end{aligned}
$$

Here $\alpha$ and $\gamma$ are the absorption coefficient and fractional coupling intensity loss, respectively. $L$ is the circumference of each ring, and $\phi$ is the combine of linear and nonlinear phase shift. $\kappa_{1}$ is the coupling coefficient, and $k=\frac{2 \pi}{\lambda}$ is the 


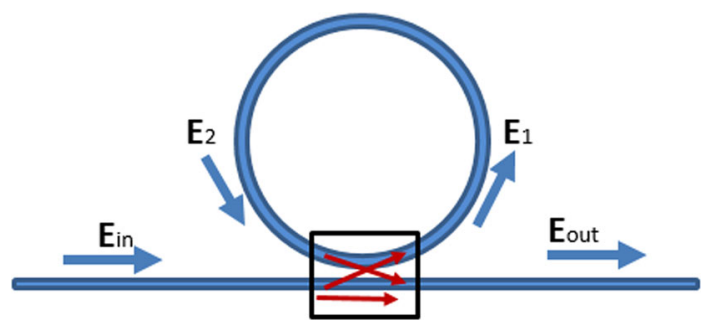

Fig. 1 Schematic diagram of an optical fiber ring resonator with a single fiber coupler

wave propagation number in a vacuum. $\tau$ is a one roundtrip loss.

The transfer function of this configuration is derived by the scattering matrix method $[20,21]$. The light in the ring resonator filter is incorporated in the attenuation constant, and the interaction can be described. The output electric field can be calculated via scattering matrix method. The proposed system decomposes the transform-limited laser pulse into the spectral domain and modified the pulse spectrum using a one-dimensional fixed or programmable spatial mask or hologram. The modified temporal spectrums are then recombined into the time domain. The input optical field in the form of Gaussian pulse can be expressed as [22]:

$E_{\text {in }}=A_{i} \exp \left[\frac{z}{2 L_{\mathrm{d}}}-i \omega_{0} t\right]$

where the amplitude of optical field is represented by $A_{i}$. The propagation distance is demonstrated by $z$. The propagation time for Gaussian pulse moves at the group velocity in a frame is $T=t-\beta_{1} \times z$. Here, $\mathrm{t}$ is the Gaussian phase shift time, and $\omega_{0}$ is the frequency shift of the Gaussian. $L_{\mathrm{d}}=T_{0}^{2} /\left|\beta_{2}\right|$ represents the dispersion length of the Gaussian pulse, where $T_{0}$ shows Gaussian pulse propagation time at the initial input. The coefficients of the linear and the second-order terms of the Taylor's expansion of the propagation constant are $\beta_{1}$ and $\beta_{2}$, respectively. For the Gaussian pulse in the microring device, a balance should be achieved between the dispersion length $\left(L_{\mathrm{d}}\right)$ and the nonlinear length $L_{\mathrm{NL}}=1 / \Gamma \phi_{\mathrm{NL}}$, where $\Gamma=n_{2} \mathrm{~K}_{0}$ is the length scale over which dispersive or nonlinear effects alter the beam diameter. For a Gaussian pulse, there is a balance between dispersion and nonlinear lengths; hence, $L_{\mathrm{d}}=L_{\mathrm{NL}}[23]$.

Based on the coupling coefficient of optical fiber ring resonator, a fraction of input Gaussian pulse is coupled into the OFRR. For long dispersive path, $E_{i}$ causes the nonlinearity effect built up inside the MRR due to change of the refractive index with optical power. Here, the power dependence of refractive index is responsible for the Kerr effect [24].
In this case, Gaussian beam has been used as an input pulse inside to microring resonators. The output of OFRR is given as:

$$
\begin{aligned}
E_{\mathrm{out}}= & E_{\mathrm{in}} y_{1} x_{1} \\
& +j \sqrt{\kappa_{1}} x_{1} \tau_{1} \exp \left(-j \phi_{1}\right)\left(\frac{j \sqrt{\kappa_{1}} x_{1} E_{\mathrm{in}}}{1-x_{1} y_{1} \tau_{1} \exp \left(-j \phi_{1}\right)}\right)
\end{aligned}
$$

The output power from each ring can be determined as:

$P_{t 3} \alpha\left(E_{\text {out }}\right) \cdot\left(E_{\text {out }}\right)^{*}=\left|E_{\text {out }}\right|^{2}$

The output signal parameters such as transmission, intensity, shift phase and group delay from the system are simulated using the MATLAB programming by iterative and numerical methods. Generally, the delay time can be calculated by considering the transmission in the singlering resonator system using Eq. (4).

$T=\frac{E_{\text {out }}}{E_{\text {in }}}=\left(\frac{x_{1} y_{1}-x_{1}^{2} \tau \exp (-j \phi)}{1-x_{1} y_{1} \tau \exp (-j \phi)}\right)$

The external phase shift of OFRRs can be achieved from the argument on ratio output field and input field as [3]:

$\phi=\arg \left(\frac{E_{\text {out }}}{E_{\text {in }}}\right)=-i \log \left(\frac{T}{|T|}\right)$

This equation gives boundary condition to achieve delay time pulse for the single-ring resonator. By solving this equation, the rate of radius for each waveguide can be calculated. Here, input power, coupler loss and coupler coefficient are fixed parameters based on experimental and theoretical previous work and research. Therefore, if the input power, coupler coefficient and core area are considered as fixed parameters, the regime of radius of ring can be calculated. Equation (6) depends on coupler coefficient, loss waveguide and internal phase which depends on input power, linear refractive and nonlinear refractive index. Equation (8) shows the relation between internal phase and input power.

$\phi=\phi_{L}+\phi_{\mathrm{NL}}=K n_{0} L+K L n_{2} P / A_{\mathrm{eff}}$

where the first part and second part are linear and nonlinear phase, respectively. $P$ is input power, and $A_{\mathrm{eff}}$ is the core area. Internal phase shift of OFRR is given by $\left(\phi=\omega_{0} T_{\mathrm{R}}\right)$, where $\omega_{0}$ is one of the resonance frequencies of the resonators and $T_{\mathrm{R}}$ is the transit time of the resonator. The phase sensitivity is obtained by differentiating the external phase shift [25].

$$
\begin{aligned}
& \phi^{\prime}=\frac{\mathrm{d} \phi}{\mathrm{d} \phi}= \\
& \frac{\left(1-x_{1}^{2} y_{1}^{2}\right) \tau^{2}}{1-2 x_{1} y_{1} \tau \cos \phi\left(\frac{1+\tau^{2}}{2}\right)+\tau^{2} x_{1}^{2} y_{1}^{2}+x_{1}^{2} y_{1}^{2}\left(\sin ^{2}(\phi)\left(1-\tau^{2}\right)^{2}\right)-\left(1-\tau^{2}\right)}
\end{aligned}
$$


Therefore, the group delay of the ring resonator can be achieved by radian frequency of the transfer function and is defined as:

$$
\begin{aligned}
& T_{\mathrm{D}}=\frac{\mathrm{d} \phi}{\mathrm{d} \omega}=\frac{\mathrm{d} \phi}{\mathrm{d} \phi} \frac{\mathrm{d} \phi}{\mathrm{d} \omega}=\phi^{\prime} T_{\mathrm{R}} \\
& T_{\mathrm{D}}= \\
& \frac{\left(1-x_{1}^{2} y_{1}^{2}\right) \tau^{2}}{1-2 x_{1} y_{1} \tau \cos \phi\left(\frac{1+\tau^{2}}{2}\right)+\tau^{2} x_{1}^{2} y_{1}^{2}+x_{1}^{2} y_{1}^{2}\left(\sin ^{2}(\phi)\left(1-\tau^{2}\right)^{2}\right)-\left(1-\tau^{2}\right)} \frac{n_{\mathrm{g}} L}{c}
\end{aligned}
$$

\section{Results and discussion}

In order to investigate some characteristics such as transmission, intensity, shift phase and group delay, Gaussian pulse is fed into the optical fiber ring resonator which consists of linear and nonlinear made of InGaAsP/InP. The Gaussian pulse as input pulse has center wavelength of $1.5 \mu \mathrm{m}$, pulse width of $50 \mathrm{~ns}$ and power of $8 \mathrm{~W}$. Figure 2a-d shows the transmission, intensity, shift phase and group delay versus wavelength in frame of linear for $k=0.1$. Figure $3 \mathrm{a}-\mathrm{d}$ shows the transmission, intensity, shift phase and group delay versus wavelength in frame of linear for $k=0.3$. Figures 4 and 5 also show the results for $k=0.5$ and $k=0.7$, respectively. Figure $6 \mathrm{a}-\mathrm{d}$ shows the transmission, intensity, shift phase and group delay versus wavelength in frame of nonlinear for

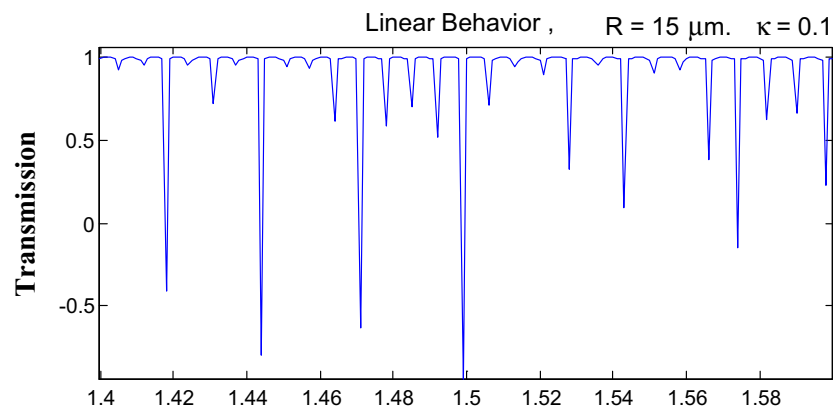

(a) Wavelength $(\mu \mathrm{m})$

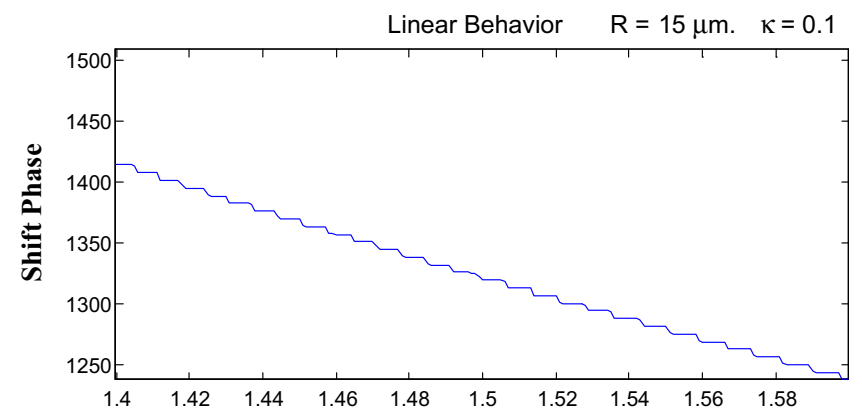

(c) Wavelength $(\mu \mathrm{m})$ $k=0.1$. Figures 7,8 and 9 show the final results for $k=0.3$, $0.5,0.7$, respectively. The fixed parameters of the system consist of radius ring resonators equal $50 \mathrm{~nm}$ and core area equals $0.5 \mu \mathrm{m}^{2}$. The roundtrip of 20,000 times inside the system produces resonance and nonlinearity. The chaotic signals because of the nonlinearity can be generated. The output power is reduced for the loss waveguide, and intensity can be enhanced and build up in some wavelengths where in some wavelength it suppress due to the resonance. The input Gaussian pulse is sliced and amplified intensity. The rate of chaotic signal depends on properties of waveguide such as radius and loss waveguide. Frequency chaotic has immense potential for applications in secure communication because of chaotic oscillators such as the universal circuit. Chaotic systems provide a potentially rich mechanism for signal design and generation for a variety of optical communications.

\section{Conclusion}

The maximum rate of shift phase and group delay has been observed in nonlinear. In frame of nonlinear, shift phase and group delay can be increased by the increase in coupling coefficient. The rate of transmission in frame of nonlinear is more than linear. In frame of nonlinear, the transmission can be increased by the decrease in coupling coefficient.

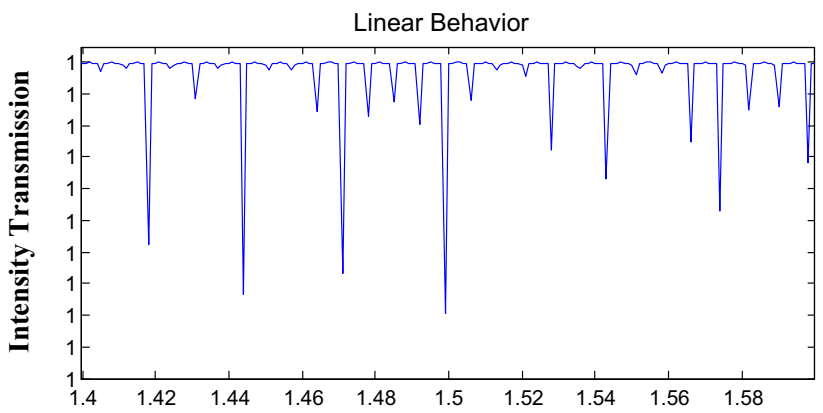

(b) Wavelength $(\mu \mathrm{m})$

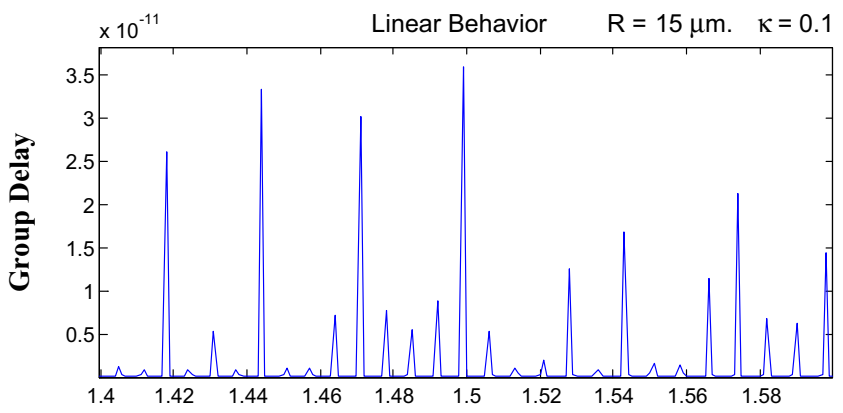

(d) Wavelength $(\mu \mathrm{m})$

Fig. 2 Linear behavior for a transmission, $\mathbf{b}$ intensity, $\mathbf{c}$ shift phase and $\mathbf{d}$ group delay for $k=0.1$ 


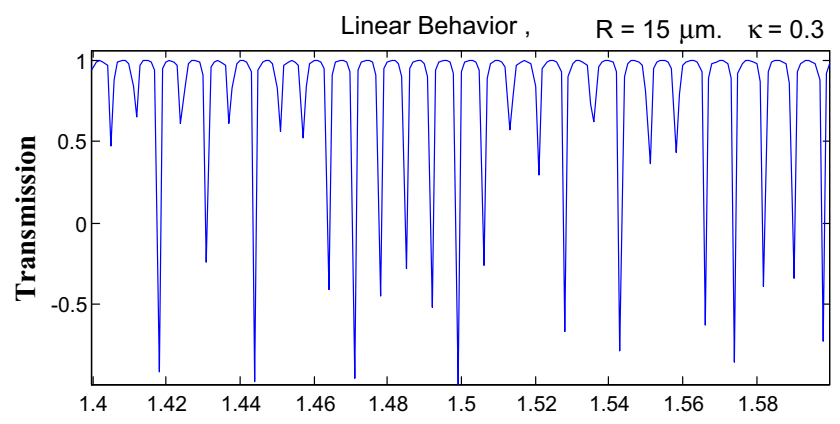

(a) Wavelength $(\mu \mathrm{m})$

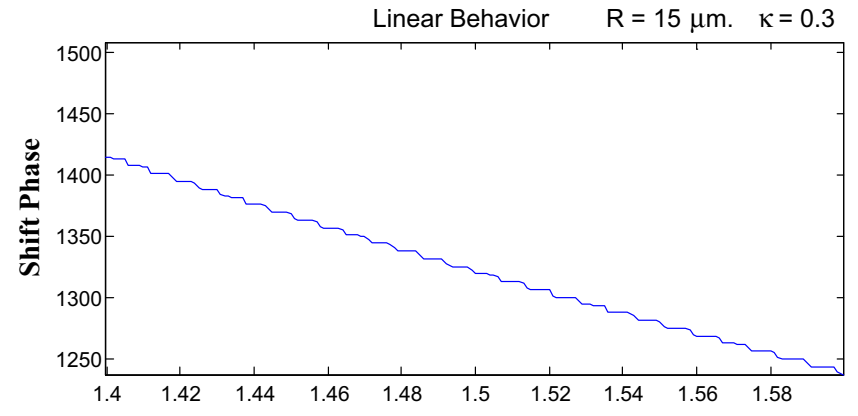

(c) Wavelength $(\mu \mathrm{m})$

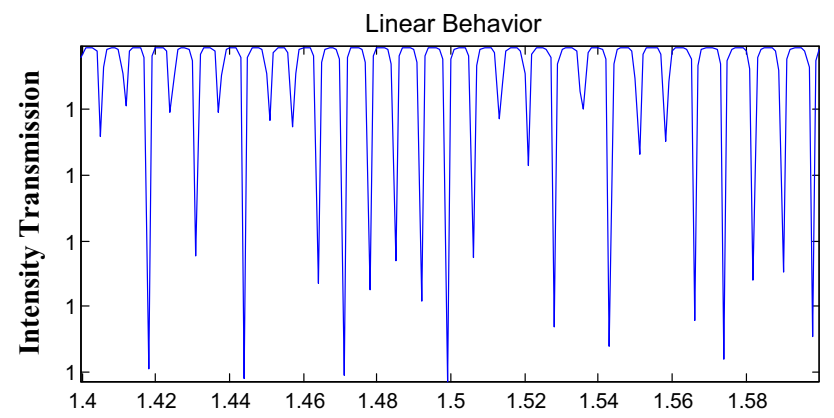

(b) Wavelength $(\mu \mathrm{m})$

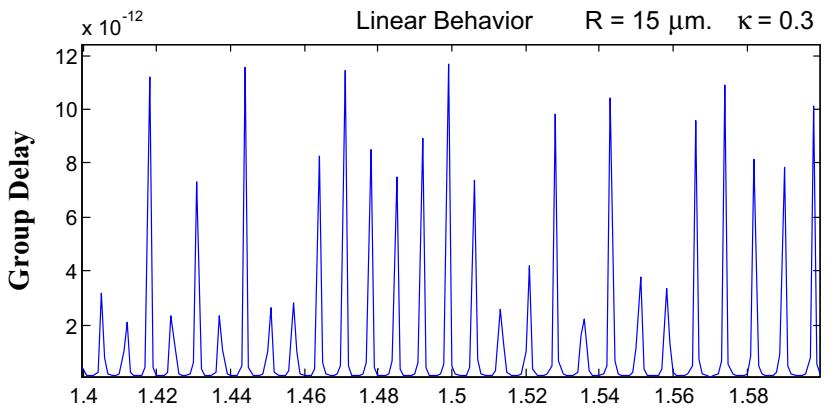

(d) Wavelength $(\mu \mathrm{m})$

Fig. 3 Linear behavior for a transmission, $\mathbf{b}$ intensity, $\mathbf{c}$ shift phase and $\mathbf{d}$ group delay for $k=0.3$

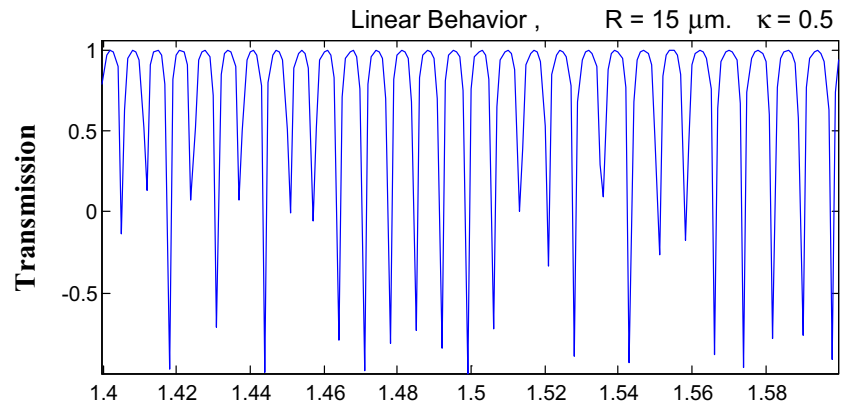

(a) Wavelength $(\mu \mathrm{m})$

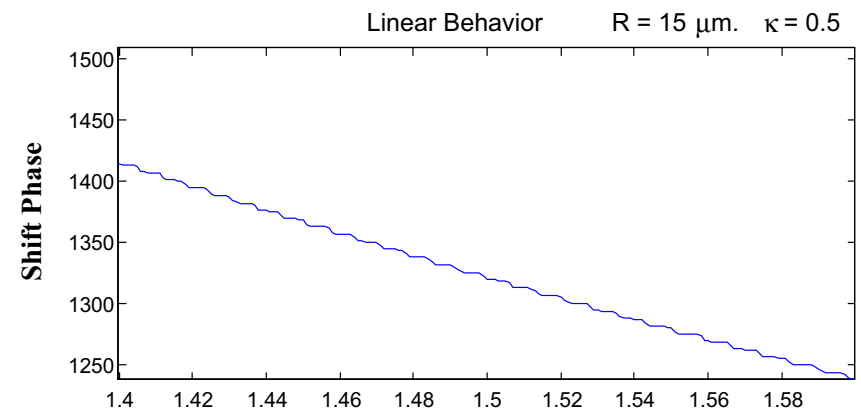

(c) Wavelength $(\mu \mathrm{m})$

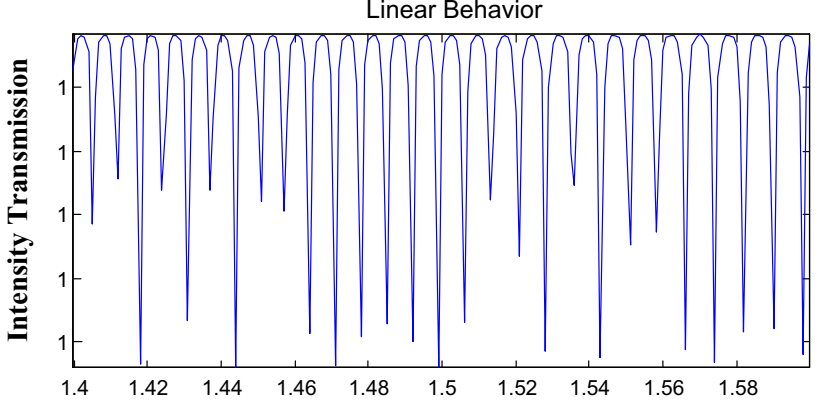

(b) Wavelength $(\mu \mathrm{m})$

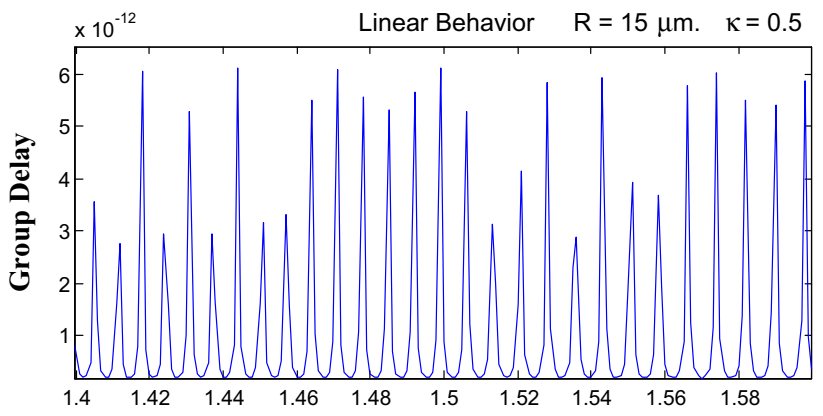

(d) Wavelength $(\mu \mathrm{m})$

Fig. 4 Linear behavior for a transmission, $\mathbf{b}$ intensity, $\mathbf{c}$ shift phase and $\mathbf{d}$ group delay for $k=0.5$ 


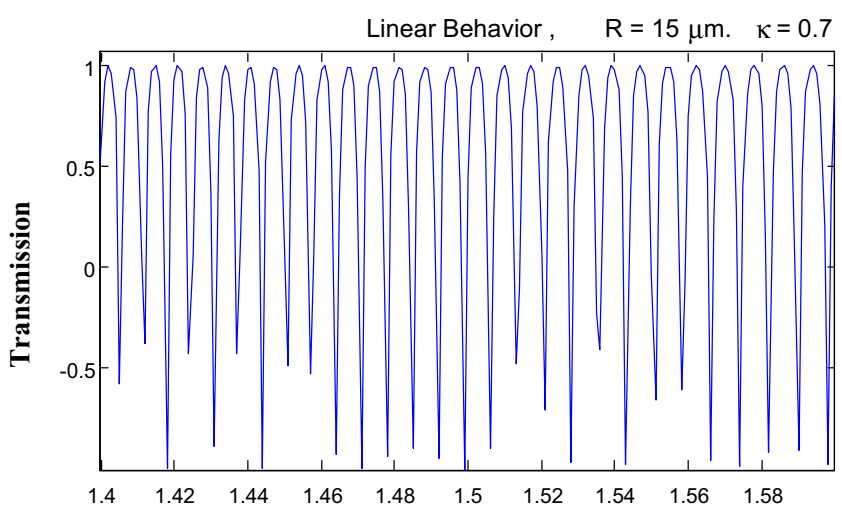

(a) Wavelength $(\mu \mathrm{m})$

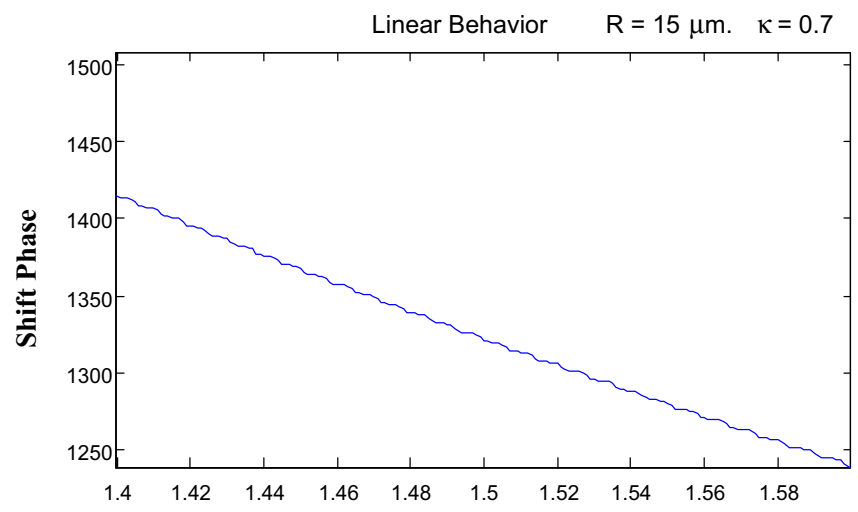

(c) Wavelength $(\mu \mathrm{m})$

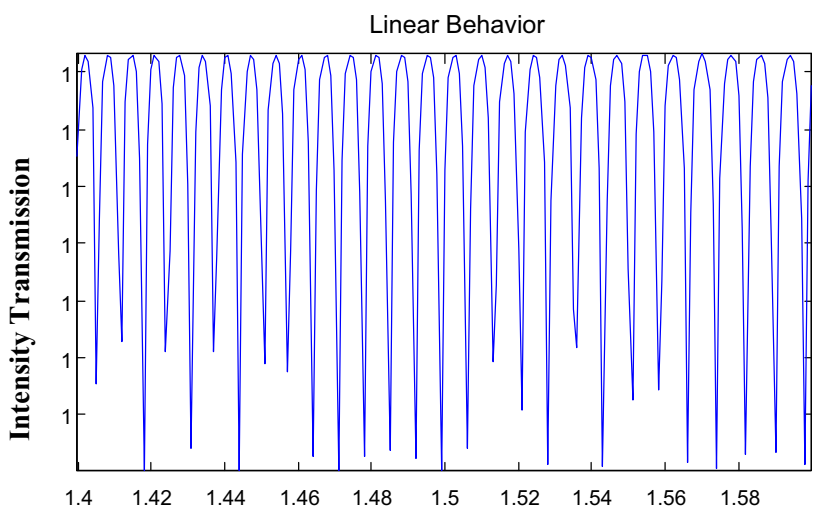

(b) Wavelength $(\mu \mathrm{m})$

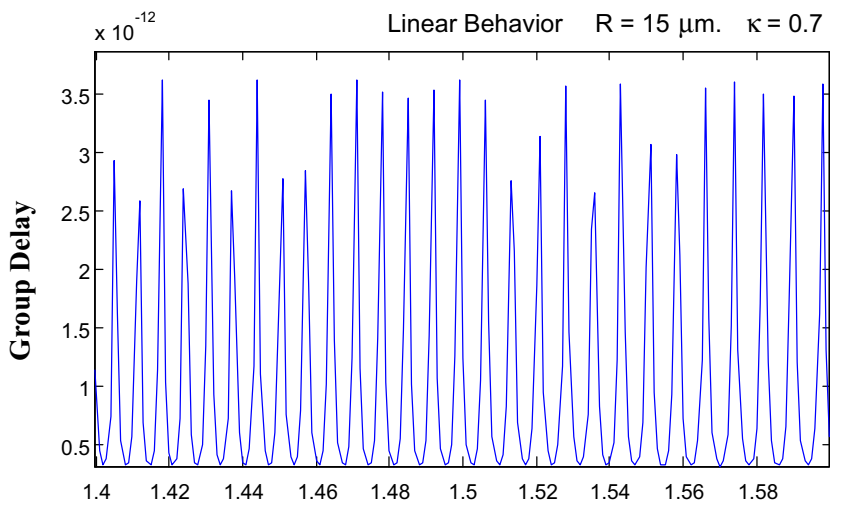

(d) Wavelength $(\mu \mathrm{m})$

Fig. 5 Linear behavior for (a) transmission, (b) intensity, (c) shift phase and (d) group delay for $\mathrm{k}=0.7$

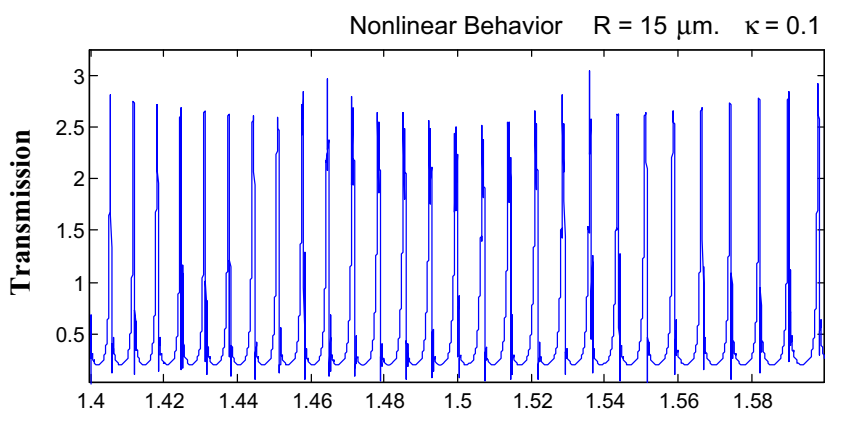

(a) Wavelength $(\mu \mathrm{m})$

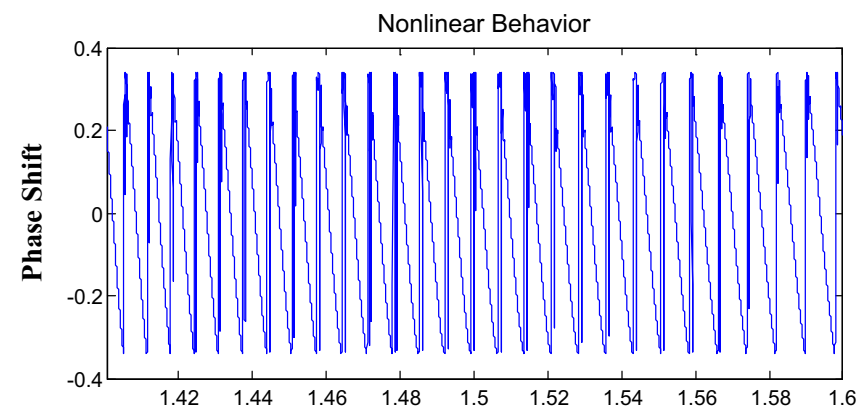

(c) Wavelength $(\mu \mathrm{m})$

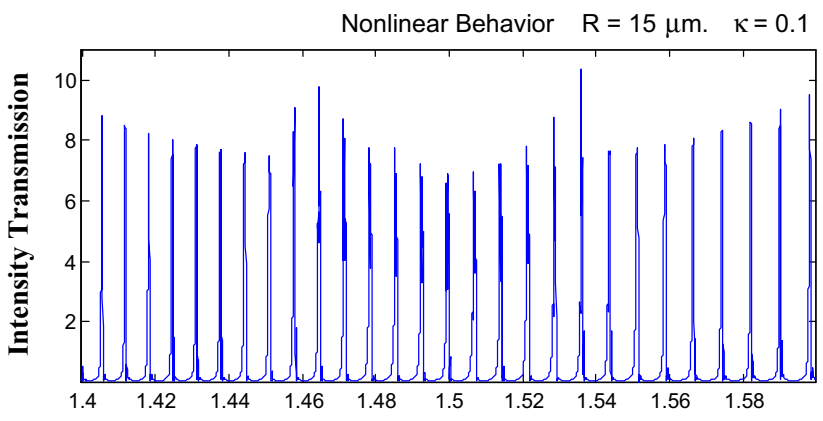

(b) Wavelength $(\mu \mathrm{m})$

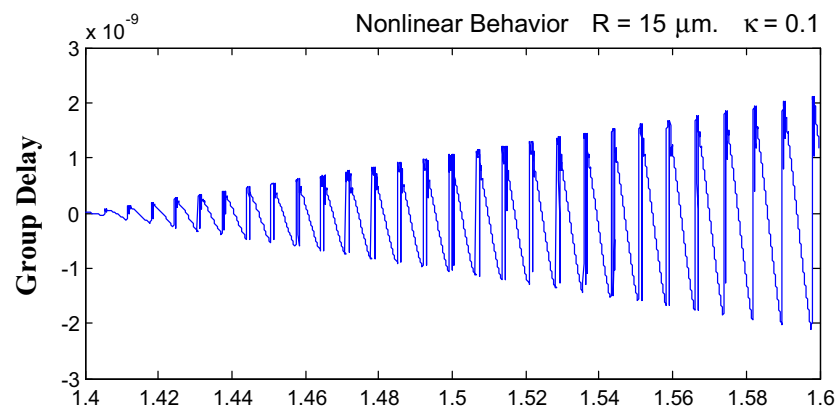

(d) Wavelength $(\mu \mathrm{m})$

Fig. 6 Nonlinear behavior for a transmission, $\mathbf{b}$ intensity, $\mathbf{c}$ shift phase and $\mathbf{d}$ group delay for $k=0.1$ 


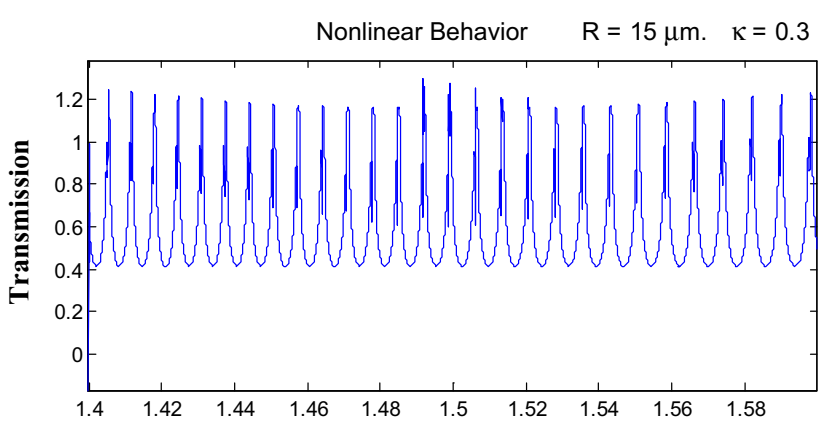

(a) Wavelength $(\mu \mathrm{m})$

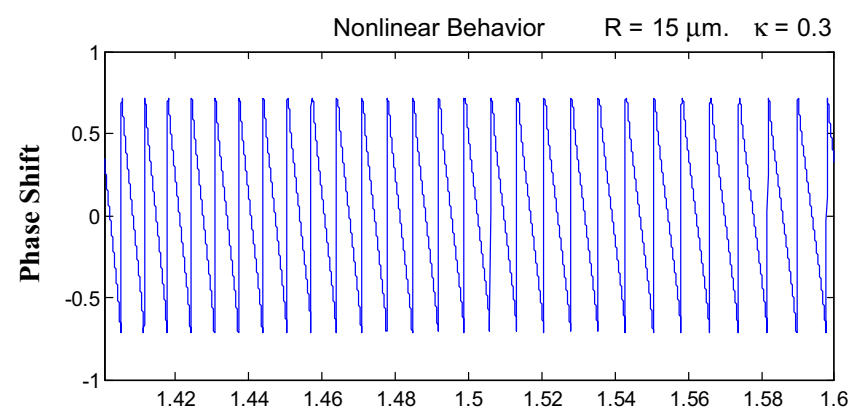

(c) Wavelength $(\mu \mathrm{m})$

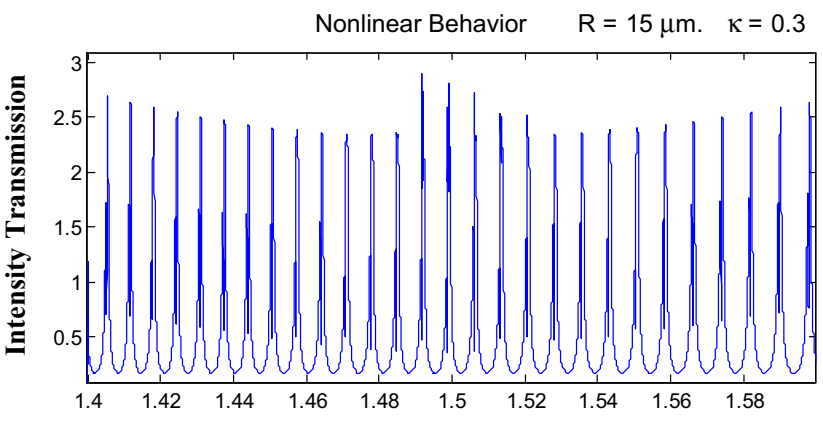

(b) Wavelength $(\mu \mathrm{m})$

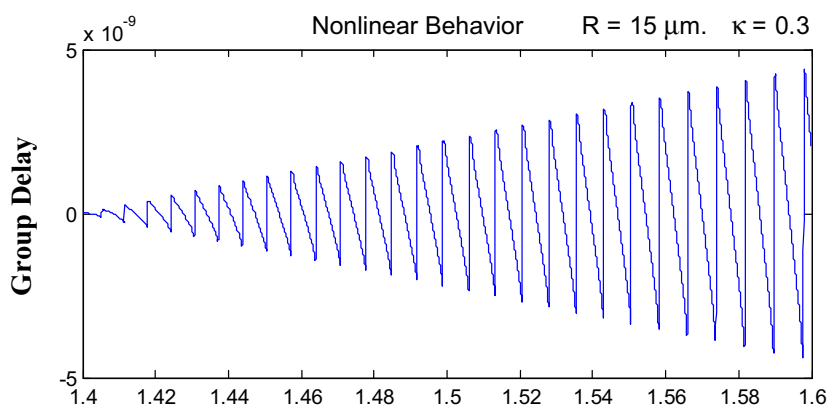

(d) Wavelength $(\mu \mathrm{m})$

Fig. 7 Nonlinear behavior for a transmission, $\mathbf{b}$ intensity, $\mathbf{c}$ shift phase and $\mathbf{d}$ group delay for $k=0.3$

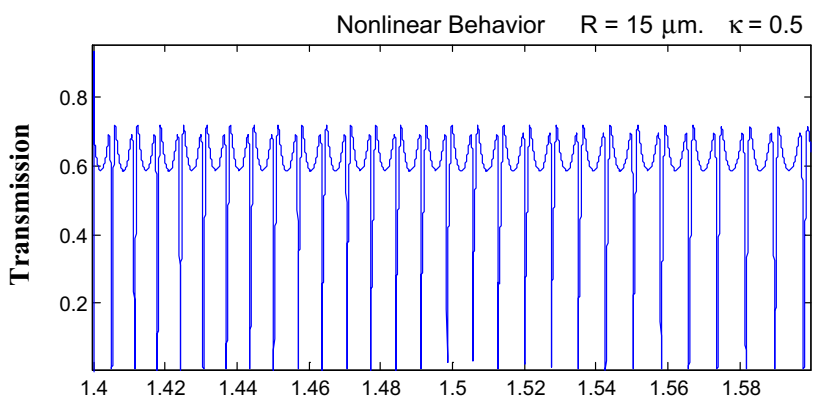

(a) Wavelength $(\mu \mathrm{m})$

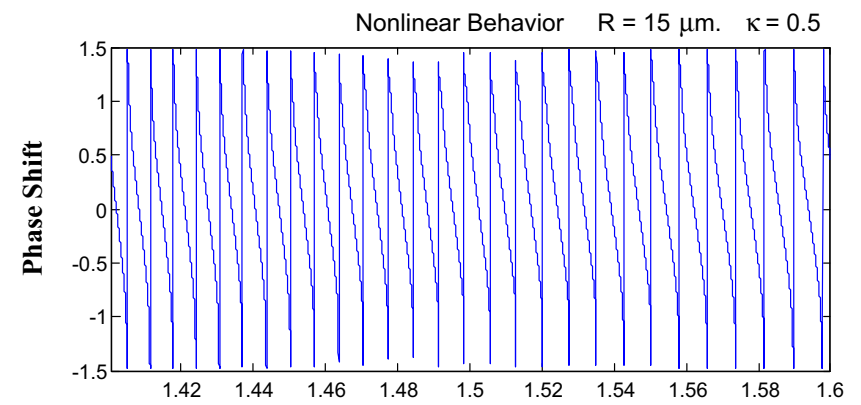

(c) Wavelength $(\mu \mathrm{m})$

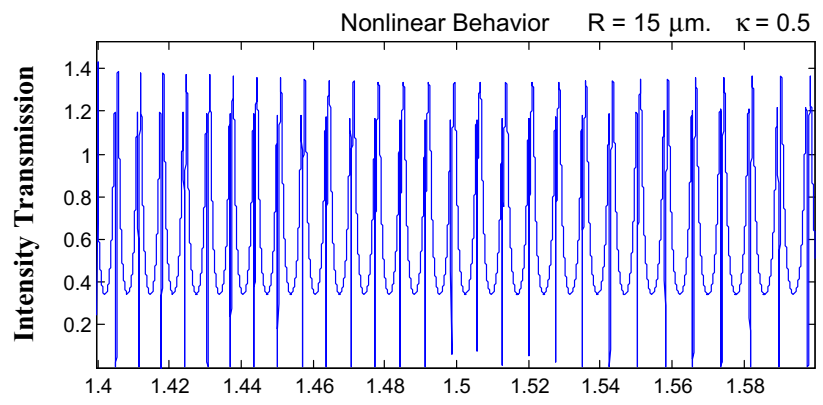

(b) Wavelength $(\mu \mathrm{m})$

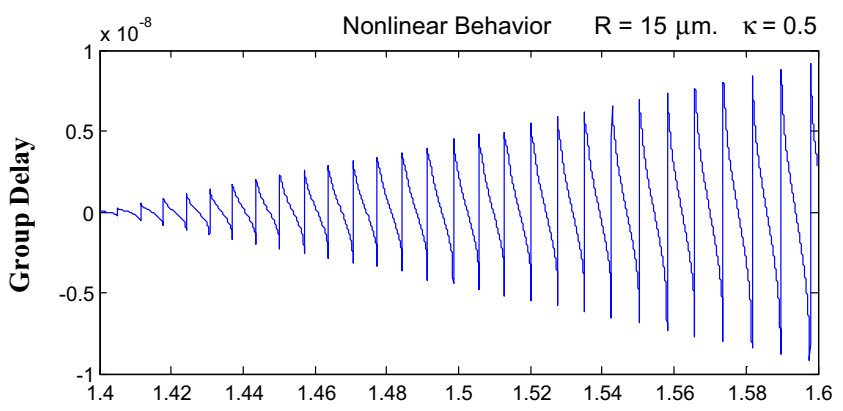

(d) Wavelength $(\mu \mathrm{m})$

Fig. 8 Nonlinear behavior for a transmission, $\mathbf{b}$ intensity, $\mathbf{c}$ shift phase and $\mathbf{d}$ group delay for $k=0.5$ 


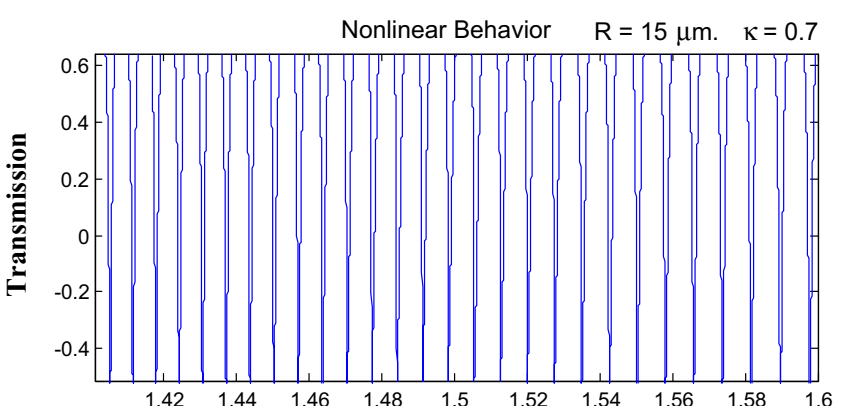

(a) Wavelength $(\mu \mathrm{m})$

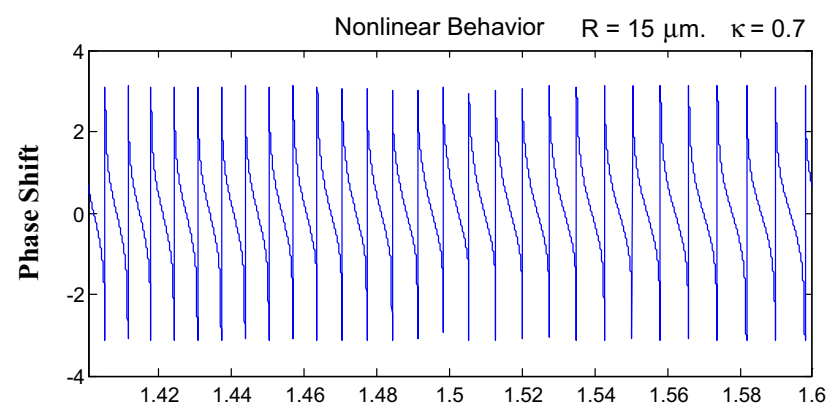

(c) Wavelength $(\mu \mathrm{m})$

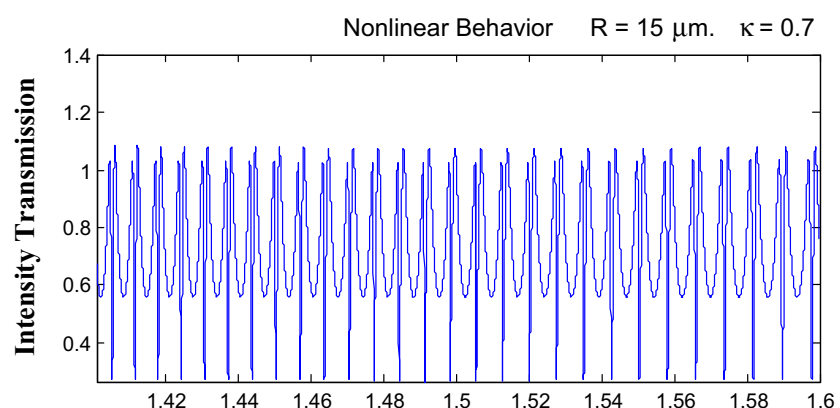

(b) Wavelength $(\mu \mathrm{m})$

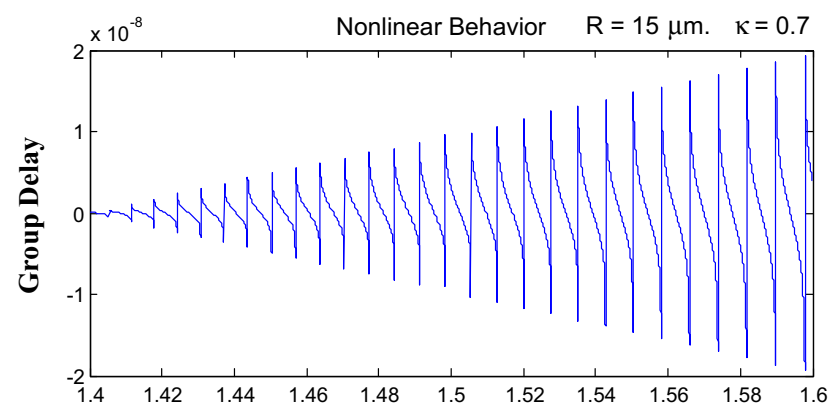

(d) Wavelength $(\mu \mathrm{m})$

Fig. 9 Nonlinear behavior for $\mathbf{a}$ transmission, $\mathbf{b}$ intensity, $\mathbf{c}$ shift phase and $\mathbf{d}$ group delay for $k=0.7$

Open Access This article is distributed under the terms of the Creative Commons Attribution 4.0 International License (http://creative commons.org/licenses/by/4.0/), which permits unrestricted use, distribution, and reproduction in any medium, provided you give appropriate credit to the original author(s) and the source, provide a link to the Creative Commons license, and indicate if changes were made.

\section{References}

1. McCall, S., et al.: Whispering-gallery mode microdisk lasers. Appl. Phys. Lett. 60(3), 289-291 (1992)

2. Madsen, C., Lenz, G.: Optical all-pass filters for phase response design with applications for dispersion compensation. IEEE Photonics Technol. Lett. 10(7), 994-996 (1998)

3. Heebner, J.E., et al.: Optical transmission characteristics of fiber ring resonators. IEEE J. Quantum Electron. 40(6), 726-730 (2004)

4. Djordjev, K., et al.: High-Q vertically coupled InP microdisk resonators. IEEE Photonics Technol.Lett. 14(3), 331-333 (2002)

5. Afroozeh, A., et al.: Simulation of soliton amplification in micro ring resonator for optical communication. J. Teknol. 55(1), 271-277 (2012)

6. Stokes, L.F., Chodorow, M., Shaw, H.J.: All-single-mode fiber resonator. Opt. Lett. 7(6), 288-290 (1982)

7. Choi, J.M., Lee, R.K., Yariv, A.: Control of critical coupling in a ring resonator-fiber configuration: application to wavelength-selective switching, modulation, amplification, and oscillation. Opt. Lett. 26(16), 1236-1238 (2001)

8. Afroozeh, A. et al.: Optical dark and bright soliton generation and amplification. In: 2010 International Conference on Enabling Science and Nanotechnology (ESciNano). IEEE (2010)
9. Heebner, J.E., Boyd, R.W.: Enhanced all-optical switching by use of a nonlinear fiber ring resonator. Opt. Lett. 24(12), 847-849 (1999)

10. Lenz, G., et al.: Optical delay lines based on optical filters. IEEE J. Quantum Electron. 37(4), 525-532 (2001)

11. Afroozeh, A., et al.: $\mathrm{THz}$ frequency generation using Gaussian pulse for medical applications. Opt. Int. J. Light Electron Opt. 124(5), 416-419 (2013)

12. Afroozeh, A., et al.: High-terahertz-frequency carrier generation by optical pulse for radio-over-fiber applications. Opt. Eng. 50(12), 125005-125005-11 (2011)

13. Boyd, R.W., Heebner, J.E.: Sensitive disk resonator photonic biosensor. Appl. Opt. 40(31), 5742-5747 (2001)

14. Afroozeh, A., et al.: Determination of Fwhm for solition trapping. J. Teknol. 55(1), 77-83 (2012)

15. Little, B.E., et al.: Microring resonator channel dropping filters. J. Lightwave Technol. 15(6), 998-1005 (1997)

16. Afroozeh, A., et al.: Comparison of control light using KramersKronig method by three waveguides. J. Comput. Theor. Nanosci. (CTN) 12(8), 1864-1868 (2015)

17. Aitchison, J., et al.: Observation of spatial solitons in AlGaAs waveguides. Electron. Lett. 28(20), 1879-1880 (1992)

18. Narayanan, K., Preble, S.F.: Optical nonlinearities in hydrogenated-amorphous silicon waveguides. Opt. Express 18(9), 8998-9005 (2010)

19. Grover, R., et al.: Vertically coupled GaInAsP-InP microring resonators. Opt. Lett. 26(8), 506-508 (2001)

20. Capmany, J., Muriel, M.A.: A new transfer matrix formalism for the analysis of fiber ring resonators: compound coupled structures for FDMA demultiplexing. J. Lightwave Technol. 8(12), 1904-1919 (1990)

21. Choi, J.M., Lee, R.K., Yariv, A.: Ring fiber resonators based on fused-fiber grating add-drop filters: application to resonator coupling. Opt. Lett. 27(18), 1598-1600 (2002) 
22. Billam, T., Cornish, S., Gardiner, S.: Realizing bright-matterwave-soliton collisions with controlled relative phase. Phys. Rev. A 83(4), 041602 (2011)

23. Bogaerts, W.: Optical interconnects. In: Advanced Interconnects for ULSI Technology, pp. 503-542. Wiley (2012)

24. Sizmann, A., Leuchs, G.: V The optical kerr effect and quantum optics in fibers. Prog. Opt. 39, 373-469 (1999)
25. Su, Y. et al.: System performance of slow-light buffering and storage in silicon nano-waveguide. In: Asia Pacific Optical Communications. International Society for Optics and Photonics (2007)

Publisher's Note Springer Nature remains neutral with regard to jurisdictional claims in published maps and institutional affiliations. 